Pacific Journal of Mathematics

CONJUGACY CLASS STRUCTURE OF SMOOTH HYPERBOLIC
SECTORS

Marcy Mason Barge, Richard Swanson
and Russell Bruce WaLker 


\title{
CONJUGACY CLASS STRUCTURE OF SMOOTH HYPERBOLIC SECTORS
}

\author{
Marcy M. Barge, Richard C. Swanson ANd Russell B. Walker
}

In this article we exhibit a continuum of explicit, cubic plus flat, nonconjugate hyperbolic sectors [Theorem 3.1]. We further show that, regardless of conjugacy class, any flat vector field with a hyperbolic sector can be locally $C^{\infty}$ approximated by a conjugate of a linear model; however, there exist non-flat hyperbolic sectors which can be arbitrarily $C^{\infty}$-approximated by linear conjugates, but are not conjugate to any of them [Theorem 5.2].

In addition, we address the following:

Problem. Which smooth maps can be realized as the pass-by or "sojourn time" map of some hyperbolic sector?

Such sojourn time maps must go to $\infty$ at zero; the asymptotic behavior at zero determines the sector's conjugacy class. We prove that if $\tau:(0,1) \rightarrow \mathbf{R}^{+}$is a smooth map such that $1 / \tau$ is smoothly extendible to zero, then there is a smooth hyperbolic sector with $\tau$ as sojourn time map [Theorem 3.1]. In other results, the variation in successive oscillations of $\tau$ provide sufficient conditions for the realization of $\tau$ as a sojourn time map [Theorems 2.1 and 4.2].

These results greatly extend previous work. F. Dumortier gave sufficient conditions for local conjugacy (via a time preserving homeomorphism) of two smooth flows with hyperbolic sectors [D1]. Later in [SSW], a first example appears of a smooth flow with hyperbolic sector which is not conjugate to a linear model. This construction involves meticulous surgery on the sector domain.

We make two related conjectures: Call the conjugates of linear models the central class. Then the Hartman-Grobman Theorem [H] guarantees that the central class has interior in the $C^{1}$ topology. Vector fields $C^{r}$-interior to the central class are $C^{r}$-structurally stable under time conjugacy. Our evidence supports the converse in the $C^{\infty}$. topology:

Conjecture A. The collection of time conjugacy stable hyperbolic sectors comprises, in the $C^{\infty}$ topology, the interior of the central class.

Finally, do the $C^{\infty}$-flat sectors represent all the conjugacy classes?

Conjecture B. Every time conjugacy class of $C^{\infty}$ hyperbolic sectors contains a vector field which is $C^{\infty}$-flat at the singularity. 
1. Statement of results. Assume $\Sigma_{1}$ and $\Sigma_{2}$ are two fixed $C^{\infty}$-smooth transversals to a $C^{\infty}$-smooth flow $\phi_{t}$, having a hyperbolic sector (Hartman $[\mathbf{H}]$ ) at the singularity $p$ (see $\S 5$ for more precise definitions). The flow ingresses the sector on $\Sigma_{1}$ and egresses through $\Sigma_{2}$. Let $W^{s}(p)$ denote the stable manifold of $p$. The sojourn map $\tau: \Sigma_{1} \backslash W^{s}(p) \rightarrow \mathbf{R}^{+}$is defined by requiring $\phi_{\tau(q)}(q) \in \Sigma_{2}$ and $\phi_{t}(q) \notin \Sigma_{2}$ for all $0 \leq t<\tau(q)$ (see Figure 1).

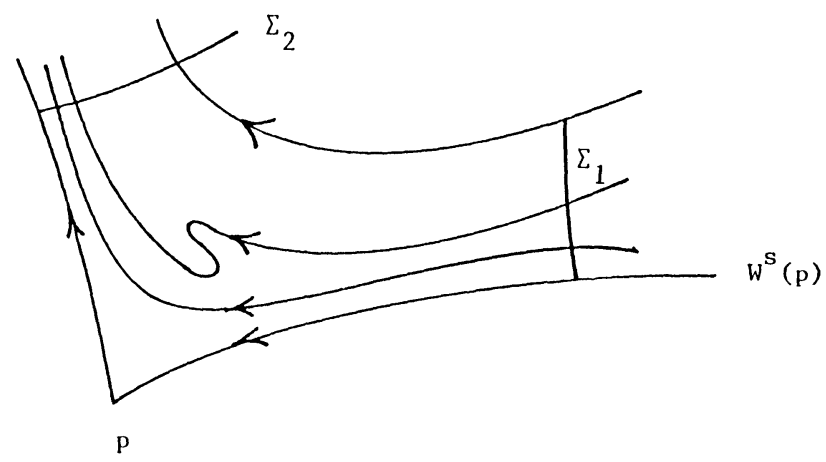

FIGURE 1

Our results concerning the realization problem depend on the sequence of time-dips and rises $\left\{\left(a_{i}, b_{i}\right)\right\}$ in the candidate $\tau$ for a sojourn map and on the degree of smoothness of $1 / \tau$ near 0 .

For now, we shall confine our attention to $C^{\infty}$-maps $\tau:(0,1) \rightarrow \mathbf{R}^{+}$ having a countable collection of ordered critical points $\left\{c_{i}\right\}_{1}^{\infty}$ such that the following hold:

(1) $\lim c_{i}=0$ as $i \rightarrow \infty$.

(2) all $c_{i}$ 's occur at local maxima or at local minima.

(3) $\tau\left(c_{1}\right)>\tau\left(c_{0}\right)$.

Let $a_{i}=\tau\left(c_{2 i-1}\right)-\tau\left(c_{2 i}\right)$ and also $b_{i}=\tau\left(c_{2 i-1}\right)-\tau\left(c_{2 i-2}\right)$. Thus, for each $i, \tau^{\prime} \geq 0$ on $\left[c_{2 i}, c_{2 i-1}\right]$ while $\tau^{\prime} \leq 0$ on $\left[c_{2 i-1}, c_{2 i-2}\right]$.

The resulting sequences $\left\{a_{i}\right\}$ and $\left\{b_{i}\right\}$ will be called, respectively, the time-dips and time-rises in $\tau(s)$ as $s \rightarrow 0+$ (see Figure 2).

If the map $\tau$ actually coincides with the sojourn map of a hyperbolic sector, then any cofinite collection of pairs $\left\{\left(a_{i}, b_{i}\right)\right\}_{i \geq n}$ determines that sector's time-conjugacy class ([SSW]). Also the collection corresponds to the standard conjugacy class unless the sequence $\left\{a_{i}\right\}$ does not tend to zero as $i \rightarrow \infty$ [SSW]. 


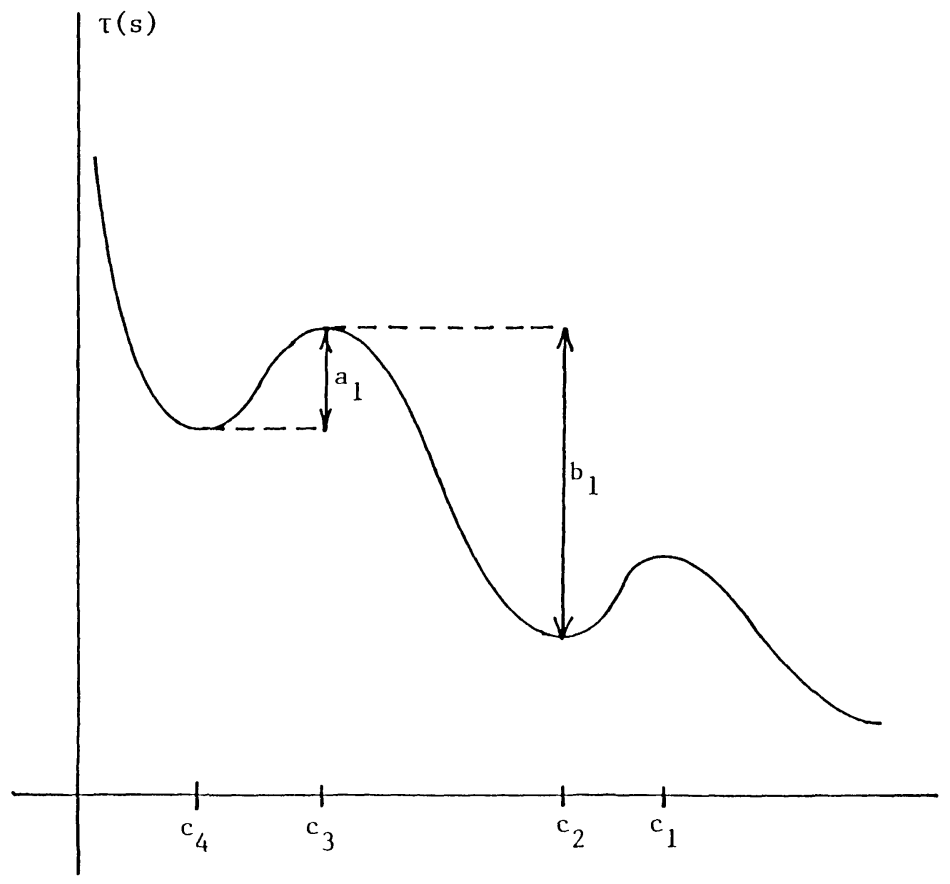

FIGURE 2

THEOREM 2.1. Given any sequence $\left\{a_{i}\right\}$ with $a_{i} \geq 0$ there exists an explicitly constructed $C^{\infty}$-smooth hyperbolic sector whose sojourn map $\tau$ realizes these $\left\{a_{i}\right\}$ as its time dips.

TheOREM 3.1. Let $\tau:(0, a) \rightarrow \mathbf{R}^{+}$be a $C^{\infty}$-map such that

(1) $\lim \tau(s)=\infty$ as $s \rightarrow 0+$, and

(2) $1 / \tau$ is $C^{\infty}$-extendible to $[0,1)$.

Then there exists a $C^{\infty}$-flow on a hyperbolic sector whose sojourn map is given by $\tau$.

THEOREM 4.2. Suppose $\left\{a_{i}\right\}$ and $\left\{b_{i}\right\}$ are sequences of positive real numbers such that, for some constant $K$,

$$
\begin{gathered}
K+\sum_{i=1}^{n}\left(b_{i}-a_{i}\right)>0, \quad \text { for all } n \text { and } \\
\sum_{n=1}^{\infty}\left\{K+\sum_{i=1}^{n}\left(b_{i}-a_{i}\right)\right\}^{-1 / p}<\infty,
\end{gathered}
$$

for all positive integers $p$. Then there is a $C^{\infty}$-hyperbolic sector whose sojourn map $\tau$ has the time-dips and rises given by the sequences $\left\{a_{i}\right\}$ and $\left\{b_{i}\right\}$. 
Conjecture C. If the vector field $X$ is $C^{\infty}$-flat and generates a hyperbolic sector, then the inverted sojourn map $1 / \tau$ is $C^{\infty}$-flat at zero.

Conjecture D. The induced sequences $\left\{a_{i}\right\}$ and $\left\{b_{i}\right\}$ of time-dips and rises in the sojourn map $\tau$ of a $C^{\infty}$-hyperbolic sector satisfy conditions (1) and (2) of Theorem 5.

Conjectures $\mathrm{A}, \mathrm{C}$, and $\mathrm{D}$, if true, would imply that every conjugacy class contains a $C^{\infty}$-flat representative that is explicitly constructible. That conclusion combined with Theorem 5.2 below would prove that every conjugacy class meets the boundary $\partial I_{0}$ of the central class.

Our main contribution to the CCCP is that the $C^{\infty}$-flat hyperbolic sectors lie on the boundary of the central class containing all the linear hyperbolic sectors.

THEOREM 5.2. If the generating vector field $X$ of a $C^{\infty}$-smooth hyperbolic sector is $C^{\infty}$-flat at the singularity, and the stable and unstable manifolds are transverse, then that sector lies on the boundary $\partial I_{0}$ of the central class. The boundary $\partial I_{0}$ also contains hyperbolic sectors that are not in $I_{0}$ and not $C^{\infty}$-flat.

REMARKs. We also know that if a sector is "straight," i.e., the orbits have at most one transversal intersection with a radial line from the origin, then that sector lies in the closure of the central class. It follows that an element in the exterior of $I_{0}-a$ candidate for a new conjugacy-stable sector-cannot be either $C^{\infty}$-flat or straight. Indeed such a hyperbolic sector cannot be polynomial or satisfy any Lojasiewicz inequality [D2].

2. Proof of Theorem 2.1. In a previous paper ([SSW]), the authors constructed a $C^{\infty}$-smooth vector field, denoted by $X_{1}$, having the following properties:

(i) $X_{1}$ has a hyperbolic sector.

(ii) $X_{1}=F \cdot X_{0}$, that is $X_{1}$ is a rescaling by a $C^{\infty}$ positive function $F(x, y)$ of the $C^{\infty}$-flat vector field

$$
X_{0}: \begin{aligned}
& x^{\prime}=-x^{3} e^{-1 / x} \\
& y^{\prime}=y^{3} e^{-1 / y}
\end{aligned}
$$

(iii) The rescaling function has the form $F=\sum_{i=1}^{\infty}\left(1+F_{i}\right)$, such that the sequence of $F_{i}$ 's have disjoint supports $P_{i}$ (as in Figure 3 ).

(iv) The sequence $\left\{\left(a_{i}, b_{i}\right)\right\}$, as defined before, corresponding to the sojourn map $\tau_{1}$ of $X_{1}$ is such that $a_{i} \geq 1$ for each $i \geq 1$. 


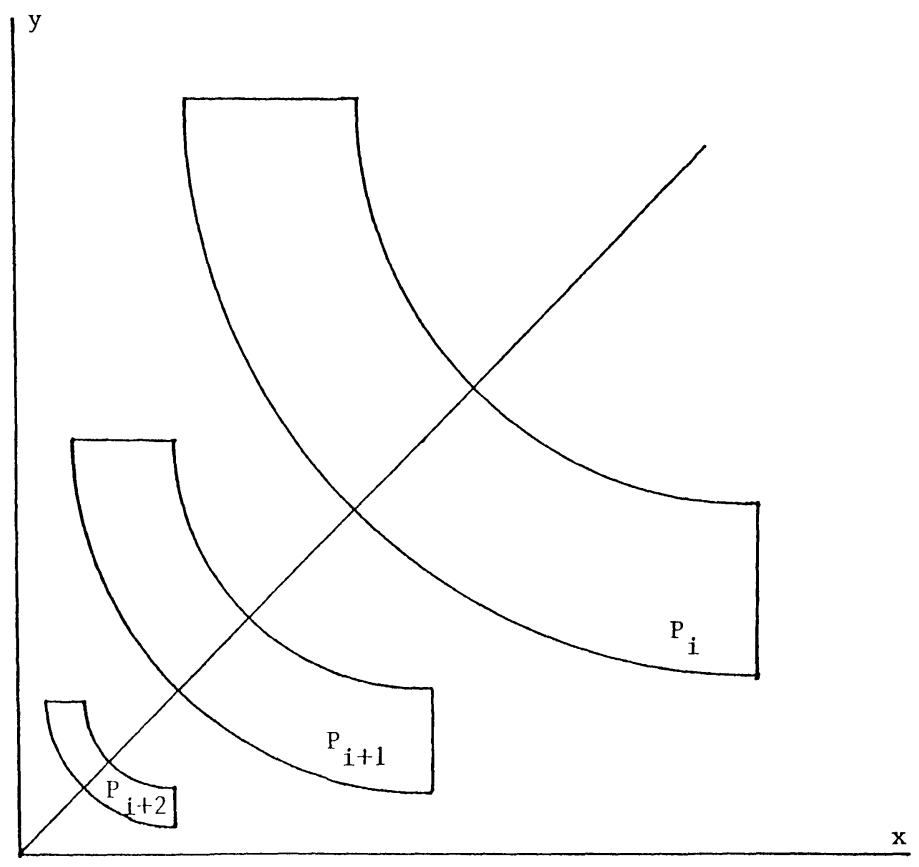

FIGURE 3

By rescaling the bump functions $F_{i}$, if necessary, employed in the construction of $X_{1}$ it is easy to create an example such that $a_{i}=1$ for all $i \geq 1$.

Note that we may further adjust any particular $F_{i}$ to equal zero without sacrificing smoothness.

Let $\left\{a_{i}^{*}\right\}$ denote the desired sequence of "dips" we need to attain in a sojourn map deriving from some rescaling $X_{*}$ of $X_{0}$, as above.

The restriction $X_{1} \mid P_{j}$ has the form $\left(1+F_{j}\right) X_{0}$, for each $j$. The sojourn times of the flow within $P_{j}$ tend to $\infty$ as $j$ increases. Thus, given $a_{k}^{*}$, there exist $m_{j k}$, for all $j$ sufficiently large, such that $\left(1+m_{j k} F_{j}\right) X_{0}$ gives rise to a dip of size precisely $a_{k}^{*}$. Since $a_{k}^{*}$ corresponds to an increasingly smaller proportion of the sojourn time in $P_{j}$, as $j$ increases, the sequence $m_{j k}$ is bounded (in fact tends to zero). Suppose $m_{j k}<M_{k}$ for all $j$.

Since $X_{1}$ is $C^{\infty}$-flat at the origin, we may choose $j(k)$ such that

$$
\left\|F_{j(k)} X_{0}\right\|_{k} \leq 1 / k M_{k}
$$


for all $k \geq 1$ where $\|\cdot\|_{k}$ denotes the uniform $C^{k}$-norm on vector fields. Putting $m_{k}=m_{j(k), k}$, the vector field

$$
X_{*}=\left(1+\sum_{k=1}^{\infty} M_{k} F_{j(k)}\right) X_{0}
$$

has the desired properties.

REMARK. For further details of this construction consult [SSW]. This result will also be obtained as an easy corollary (Corollary 4.3) of the main theorem of $\S 4$.

3. Sojourn map realization: The Proof of 3.1. Let $a \neq 0$ be a constant, and suppose

$$
x^{\prime}=-x\left[a^{2}+\left(\frac{x-y}{2}\right)^{2}\right], \quad y^{\prime}=y\left[a^{2}+\left(\frac{x-y}{2}\right)^{2}\right] .
$$

Then the sojourn map time to pass from the (ingress) point $(1, s)$ to the (egress) point $(s, 1)$ is given by

$$
\tau(s)=\int_{s}^{1} \frac{d x}{x\left[a^{2}+(x-s / x)^{2} / 4\right]}
$$

since $x y=s$ is constant on solution curves. Given the substitution $u=(x-y) / 2=(x-s / x) / 2$, the integral in (1) becomes

$$
\tau(s)=\int_{(s-1) / 2}^{(1-s) / 2} \frac{d u}{\left(\sqrt{u^{2}+s}\right)\left(u^{2}+a^{2}\right)} .
$$

Now replace $a^{2}$ by $s g^{2}(s)$ where $g^{2}(s)<1$. Trigonometric substitutions now yield the result

$$
\tau(s)=\left(\frac{2}{s}\right) \frac{\operatorname{Tan}^{-1}\left(\frac{1}{g} \sqrt{\left(1-g^{2}\right)(1-s) /(1+s)}\right)}{g \sqrt{1-g^{2}}} .
$$

Our aim now is to implicitly solve for $g$ as a $C^{\infty}$ function of $s$. Toward this end, we can recast (3) in the form

$$
g \sqrt{1-g^{2}}-(2 / s \tau(s)) \operatorname{Tan}^{-1}\left(\frac{1}{g} \sqrt{\left(1-g^{2}\right)(1-s) /(1+s)}\right)=0 .
$$

Suppose the left-hand side defines a function $T(s, g)$ for $0<s<1$ and $0<g<1$. Our hypotheses imply that $1 / s \tau$ is $C^{\infty}$-extendible to $[0,1)$ as $s \rightarrow 0+$. Also, if the inverse tangent is defined to be $\pi / 2$ when $g=0$, then $T(s, g)$ is $C^{\infty}$-extendible to $[0,1) \times[0,1)$. Now extend 
$T(s, g)$ to $(-1,1) \times(-1,1)$ in an arbitrary, but $C^{\infty}$, manner. This is possible by a theorem due to Borel (e.g. see [GG], p. 98). Suppose that $\lim _{s \rightarrow 0}(2 / s \tau(s))=a_{0} \geq 0$. If $a_{0}<1$, the intermediate value theorem shows there exists a number $0 \leq g_{0}<1$ such that $T\left(0, g_{0}\right)=0$. A simple computation shows that $(\partial T) / \partial g\left(0, g_{0}\right) \neq 0$.

The implicit function theorem implies that there is a $\delta>0$ and a $C^{\infty}$ function $g(s)$ defined for $|s|<\delta$ such that

$$
T(s, g(s))=0
$$

for $|s|<\delta$. It also follows that $g(s)<1$ for $|s|<\delta$. Thus, the system

$$
X_{g}: \begin{aligned}
& x^{\prime}=-x\left(s g^{2}(s)+((s-y) / 2)^{2}\right) \\
& y^{\prime}=y\left(s g^{2}(s)+((x-y) / 2)^{2}\right)
\end{aligned}
$$

has a sojourn map $\tau_{g}$ which equals $\tau$ for $|s|<\delta$.

If $a_{0} \geq 1$, then the map $\sigma=2 a_{0} \tau$ can be realized in the above fashion as a sojourn map of some $X_{g}$ since

$$
\lim _{s \rightarrow 0^{+}}\left(\frac{2}{s \sigma(s)}\right)=\frac{1}{2}<1 .
$$

To realize $\tau(s)$ itself, use the vector field $\left(1 / 2 a_{0}\right) X_{g}$. Again, this is valid for $s<\delta$.

To realize $\tau(s)$ on $\delta \leq s<1$, first extend $g(s)$ in a $C^{\infty}$-manner so that $g(s) \neq 0$ on $\delta \leq s<1$. This smoothly extends the vector field $X_{g}$, still denoted by $X_{g}$. Let $\tau_{g}$ denote the sojourn map on $(0,1)$ for $X_{g}$. The function $\tau_{g}(s) / \tau(s)$ is $C^{\infty}$ on $[0,1)$ and is identically 1 for $s<\delta$. Define $Y=\left(\tau_{g}(x y) / \tau(x y)\right) \cdot X_{g}$. Since $x y=s$ is constant, the vector field $Y$ has sojourn map $\tau_{Y}=\left(\tau / \tau_{g}\right) \cdot \tau_{g}=\tau$ for $0<s<1$.

\section{Realizing rises and dips in the $\tau$-map.}

LeMmA 4.1. Let $f:[0,1] \rightarrow \mathbf{R}^{+} \cup\{0\}$ be a $C^{\infty}$ function with $f(0)=0$ and $f(x)>0$ for $x>0$. Suppose that: $f$ has local extrema at $c_{0}=$ $1>c_{1}>c_{2}>\cdots ; f\left(c_{2 i}\right)$ is a local max, $f\left(c_{2 i+1}\right)$ is a local min, and $f$ is monotone on $\left[c_{i+1}, c_{i}\right]$ for $i=0,1,2, \ldots$; and $c_{i} \rightarrow 0$ as $i \rightarrow \infty$. Let $\bar{a}_{i}=f\left(c_{2 i}\right)-f\left(c_{2 i-1}\right)$ and $\bar{b}_{i}=f\left(c_{2 i-2}\right)-f\left(c_{2 i-1}\right)$ for $i=1,2, \ldots$. Then:

(i) $\sum_{i=1}^{\infty}\left(\bar{b}_{i}-\bar{a}_{i}\right)>0, \sum_{i=1}^{\infty}\left(\bar{b}_{i}-\bar{a}_{i}\right)>\bar{b}_{n+1}+\sum_{i=1}^{n}\left(\bar{b}_{i}-\bar{a}_{i}\right)$ for all $n \geq 1$, and

(ii) $\sum_{i=1}^{\infty}\left(\bar{b}_{i}\right)^{1 / p}<\infty$ and $\sum_{i=1}^{\infty}\left(\bar{a}_{i}\right)^{1 / p}<\infty$ for all positive integers $p$. 
Conversely, if $\left\{a_{i}\right\}$ and $\left\{b_{i}\right\}$ are sequences of positive integers satisfying (i) and (ii) then there is a function $f$ and a sequence of points $c_{0}=1>c_{1}>\cdots>0$ satisfying the above hypotheses such that $\bar{a}_{i}=f\left(c_{2 i}\right)-f\left(c_{2 i-1}\right)$ and $\bar{b}_{i}=f\left(c_{2 i-2}\right)-f\left(c_{2 i-1}\right)$.

Proof. Let $f$ be as hypothesized. Then (i) follows from the fact that $f(x)>0$ for $x>0$. Let $\Delta X_{i}=c_{i}-c_{i+1}$ for $i=0,1,2, \ldots$

We claim that for each $i \geq 0$ and positive integer $p$ there is a $z_{i}^{p} \in$ $\left[c_{i+2} p^{-1}, c_{i}\right)$ such that $f^{(p)}\left(z_{i}^{p}\right)=0$. This is clear for $p=1$ since $f^{\prime}\left(c_{i}\right)=0$ for $i=1,2, \ldots$ Also, if $z_{i+2}^{p} p-1 \in\left[c_{i+2} p, c_{i+2} p-1\right)$ and $z_{i}^{p} \in\left[c_{i+2} p-1, c_{i}\right)$ are such that $f^{(p)}\left(z_{i+2}^{p} p-1\right)=f^{(p)}\left(z_{i}^{p}\right)=0$, then, by the mean value theorem, there is a $z_{i}^{p+1} \in\left[z_{i+2}^{p} p-1, z_{i}^{p}\right] \subseteq\left[c_{i+2}, c_{i}\right)$ such that $f^{(p+1)}\left(z_{i}^{p+1}\right)=0$. Induction on $p$ establishes the claim.

Now we claim that for each $i \geq 0$ and positive integer $p$ there is a point $\eta_{i}^{p} \in\left[c_{i+2} p-1, c_{i}\right]$ such that

$$
\frac{\left|f\left(c_{i+1}\right)-f\left(c_{i}\right)\right|}{\left(\Delta X_{i}+\cdots+\Delta X_{i+2} p-1\right)^{p}} \leq\left|f^{(p)}\left(\eta_{i}^{p}\right)\right| .
$$

For $p=1$ this is just the mean value theorem. Assume that the claim is true for some positive $p$ for all $i \geq 0$. Now $\left|f^{(p)}(x)\right|$ takes on values $\left|f^{(p)}\left(\xi_{i}^{p}\right)\right|=0$ and

$$
\left|f^{(p)}\left(\eta_{i}^{p}\right)\right| \geq \frac{\left|f\left(c_{i+1}\right)-f\left(c_{i}\right)\right|}{\left(\Delta X_{i}+\cdots+\Delta X_{i+2} p-1\right)}
$$

on the interval $\left[c_{i+2} p-1, c_{i}\right]$. Thus there is a point $\eta_{i}^{p+1} \in\left[c_{i+2} p-1, c_{i}\right]$ such that

$$
\begin{aligned}
\left|f^{(p+1)}\left(\eta_{i}^{p+1}\right)\right| & \geq \frac{\left|f\left(c_{i+1}\right)-f\left(c_{i}\right)\right|}{\left(\Delta X_{i}+\cdots+\Delta X_{i+2} p-1\right)^{p}\left(\Delta X_{i}+\cdots+\Delta X_{i+2} p-1\right)} \\
& \geq \frac{\left|f\left(c_{i+1}\right)-f\left(c_{i}\right)\right|}{\left(\Delta X_{i}+\cdots+\Delta X_{i+2} p\right)^{p}} .
\end{aligned}
$$

Since $z_{i}^{p} \rightarrow 0$ as $i \rightarrow \infty$ for each $p$ and $f$ is $C^{\infty}, f^{(p)}(0)=0$ for all $p$. Thus, $f^{(p)}\left(\eta_{i}^{p}\right) \rightarrow 0$ as $i \rightarrow \infty$ for each $p$ and we have:

$$
\frac{\bar{a}_{i}}{\left(\Delta X_{2 i-1}+\cdots+\Delta X_{2 i-1+2} p-1\right)^{p}} \leq\left|f^{(p)}\left(\eta_{2 i-1}^{p}\right)\right| \rightarrow 0
$$

as $i \rightarrow \infty$ for each $p$. It follows that, for each positive integer $p$, there is a $K_{p}<\infty$ such that

$$
\left(\bar{a}_{i}\right)^{1 / p} \leq K_{p}\left(\Delta X_{2 i-1}+\cdots+\Delta X_{2 i-1+2} p-1\right)
$$


for all $i \geq 1$. Thus,

$$
\sum_{i=1}^{\infty}\left(\bar{a}_{i}\right)^{1 / p} \leq\left(K_{p}\right) 2^{p} \sum_{i=0}^{\infty} \Delta X_{i}=2^{p} K_{p}<\infty
$$

Similarly, $\sum_{i=1}^{\infty}\left(\bar{b}_{i}\right)^{1 / p}<\infty$ for all positive integers $p$ and we have established (ii).

For the converse, let $\left\{\bar{a}_{i}\right\}$ and $\left\{\bar{b}_{i}\right\}$ be sequences of positive real numbers satisfying (i) and (ii). Let $g: \mathbf{R} \rightarrow \mathbf{R}$ have the properties: $g$ is $C^{\infty}$ and $g(x)=0$ for $|x| \geq 1 ; g(0)=1$ and $g^{(p)}(0)=0$ for all $p \geq 1 ; g$ is monotone on $[-1,0]$ and on $[0,1]$. Also, let $L=$ $\sum_{n=1}^{\infty} b_{n}-\sum_{n=1}^{\infty} a_{n}>0$.

Given a sequence $c_{0}=1>c_{1}>c_{2}>\cdots>0$ with $c_{i} \rightarrow 0$ as $i \rightarrow \infty$, define $f:[0,1] \rightarrow \mathbf{R}$ by

$$
f(x)=\left\{\begin{array}{c}
L-\left(\sum_{i=1}^{n+1} \bar{b}_{i}-\sum_{i=1}^{n} \bar{a}_{i}\right)+\bar{b}_{n+1} g\left(\frac{x-c_{2 n}}{c_{2 n}-c_{2 n+1}}\right), \\
\text { for } x \in\left[c_{2 n+1}, c_{2 n}\right], n=0,1, \ldots ; \\
L-\left(\sum_{i=1}^{n+1} \bar{b}_{i}-\sum_{i=1}^{n} \bar{a}_{i}\right)+\bar{a}_{n+1} g\left(\frac{x-c_{2 n+2}}{c_{2 n+1}-c_{2 n+2}}\right), \\
\text { for } x \in\left[c_{2 n+2}, c_{2 n+1}\right], n=0,1, \ldots ; \\
0, \quad \text { for } x=0 .
\end{array}\right.
$$

Then $f$ is $C^{\infty}$ on $(0,1]$, continuous at $x=0$, and $f(x)=0$ if and only if $x=0$. Also, $f$ has local maxima at $c_{2 i}$, local minima at $c_{2 i-1}$, and $f$ is monotone on $\left[c_{i}, c_{i-1}\right]$ for $i=1,2, \ldots$. Thus we need only choose the $c_{i}$ such that $f$ is $C^{\infty}$ at $x=0$.

Let $M_{1}=1$ and, for $p \geq 2$,

$$
M_{p}=\max \left\{1+M_{p-1}, \text { inf }\left\{m \geq 1 \mid \sum_{i=m}^{\infty}\left(\bar{b}_{i}\right)^{1 / p} \leq 1 /\left(p^{2}\right)\right\}\right\} .
$$

For $M_{p}<i<M_{p+1}$ define $\Delta X_{2 i-1}$ by $\Delta X_{2 i-1}=\left(1 / K_{1}\right)\left(\bar{b}_{i}\right)^{1 / p}$ where $K_{1}$ is chosen so that $\sum_{i=1}^{\infty} \Delta X_{2 i-2}=1 / 2$. Similarly, let $N_{1}=1$ and, for $p \geq 2$,

$$
N_{p}=\max \left\{1+N_{p-1}, \inf \left\{m \geq 1 \mid \sum_{i=1}^{\infty}\left(a_{i}\right)^{1 / p} \leq 1 /\left(p^{2}\right)\right\}\right\} .
$$

For $N_{p} \leq i<N_{p+1}$ define $\Delta X_{2 i}$ by $\Delta X_{2 i}=\left(1 / K_{2}\right)\left(a_{i}\right)^{1 / p}$ where $K_{2}$ is chosen so that $\sum_{i=1}^{\infty} \Delta X_{2 i}=1 / 2$. 
It is an easy matter to check that $\bar{a}_{i} /\left(\Delta X_{2 i}\right)^{p} \rightarrow 0$ and $\bar{b}_{i} /\left(\Delta X_{2 i-1}\right)^{p} \rightarrow$ 0 as $i \rightarrow \infty$ for each positive integer $p$. Now let $c_{0}=1$ and $c_{i}=$ $1-\left(\sum_{j=1}^{i} \Delta X_{j}\right)$ for $i \geq 1$. With this choice of the $c_{i}$ we claim that $f$ is $C^{\infty}$ at 0 . Indeed, let $m_{p}=\max \left|g^{(p)}(x)\right|$ for $p=0,1,2, \ldots$ Then

$$
\left|f^{(p)}(x)\right| \leq m_{p} \cdot \frac{\bar{b}_{n+1}}{\left(\Delta X_{2 n+1}\right)^{p}} \quad \text { for } x \in\left[c_{2 n+1}, c_{2 n}\right]
$$

and

$$
\left|f^{(p)}(x)\right| \leq m_{p} \cdot \frac{\bar{a}_{n+1}}{\left(\Delta X_{2 n+2}\right)^{p}} \quad \text { for } x \in\left[c_{2 n+2}, c_{2 n+1}\right] .
$$

Thus, $f^{(p)}(x) \rightarrow 0$ as $x \rightarrow 0$ for all $p$ and $f$ is $C^{\infty}$ at $x=0$.

THEOREM 4.2. If $\left\{a_{i}\right\}$ and $\left\{b_{i}\right\}$ are sequences of positive real numbers such that, for some constant $K, K+\sum_{i=1}^{n}\left(b_{i}-a_{i}\right)>0$ for all $n$ and $\sum_{n=1}^{\infty}\left(K+\sum_{i=1}^{n}\left(b_{i}-a_{i}\right)\right)^{-1 / p}<\infty$ for all positive integers $p$ then there is a $C^{\infty}$ hyperbolic sector $X$ whose sojourn map $\tau_{X}$ has dips and rises given by $\left\{a_{i}\right\}$ and $\left\{b_{i}\right\}$.

Proof. Let

$$
\bar{a}_{n}=\left(K+\sum_{i=1}^{n}\left(b_{i}-a_{i}\right)\right)^{-1}-\left(K+\sum_{i=1}^{n}\left(b_{i}-a_{i}\right)+a_{n}\right)^{-1}
$$

and

$$
\bar{b}_{n}=\left(K+\sum_{i=1}^{n-1}\left(b_{i}-a_{i}\right)\right)^{-1}-\left(K+\sum_{i=1}^{n}\left(b_{i}-a_{i}\right)+a_{n}\right)^{-1} .
$$

Then $\left\{\bar{a}_{n}\right\}$ and $\left\{\bar{b}_{n}\right\}$ satisfy the conditions of Lemma 4.1. Let $f$ be the corresponding $C^{\infty}$ function given by that lemma and let $\tau=1 / f$. By Theorem 3.1 there is a $C^{\infty}$ hyperbolic sector $X$ such that $\tau_{X}=\tau$. The dips and rises of $\tau_{X}$ are then given by $\left\{a_{i}\right\}$ and $\left\{b_{i}\right\}$.

COROLlaRy 4.3. Let $\left\{a_{i}\right\}$ be any sequence of positive real numbers. Then there is a $C^{\infty}$ hyperbolic sector $X$ whose sojourn map $\tau_{X}$ realizes the sequence $\left\{a_{i}\right\}$ as its sequence of dips.

Proof. In the preceding theorem, let $b_{i}=a_{i}+2^{i}$.

5. $C^{\infty}$-flat hyperbolic sectors. In this section we prove that those hyperbolic sectors having generating vector fields which are $C^{\infty}$-flat at $(0,0)$, lie on the boundary of the central class, $I_{0}$, as determined by the $C^{\infty}$-uniform topology. This topology is now formalized. 
Our results apply to a class of "standard" $C^{\infty}$-flows, $\mathscr{H}_{0}$, on $\mathbf{R}^{2}$ having hyperbolic sectors and, in fact, to all diffeomorphic images, $\mathscr{H}$, of such flows. In either case, we first fix the location of a hyperbolic sector, then topologize the collection of $C^{\infty}$-flows having sectors in the same locale. The reader may consult Hartman $([\mathbf{H}])$ for standard terminology.

Let $\mathscr{H}_{0}$ denote the collection of $C^{\infty}$-flows $\phi_{t}$ on $\mathbf{R}^{2}$ having a singularity at $(0,0)$ and such that

(a) the stable manifold, $W^{s}(0,0)=\{(x, 0) \mid x \geq 0\}$, and the unstable manifold, $W^{u}(0,0)=\{(0, y) \mid y \geq 0\}$,

(b) the segments,

$$
\Sigma_{1}=\{(2, y) \mid 0<y \leq 1\}, \quad \Sigma_{2}=\{(x, 2) \mid 0<x \leq 1\}
$$

are differentiably transverse to the generating vector field $X$ for $\phi_{t}$,

(c) there exists $a>0$ such that corresponding to

$$
\Sigma_{1}^{a}=\{(2, y) \mid 0<y \leq a\} \subseteq \Sigma_{1},
$$

there is a well defined sojourn map, $\tau:(0, a] \rightarrow \mathbf{R}$ with $\tau(y)>0$, $\phi_{\tau(y)}(2, y) \in \Sigma_{2}$, and $\phi_{t}(2, y) \notin \Sigma_{1} \cup \Sigma_{2}$ for all $0<t<\tau(y)$ (see Figure 4).

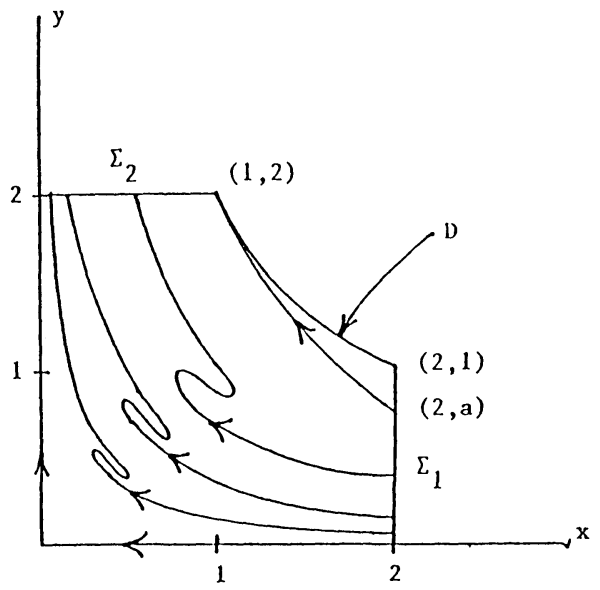

FIGURE 4

Let $D=\{(x, y) \mid 0 \leq x \leq 2,0 \leq y \leq 2, x y \leq 1\}$. The flows $\phi_{t} \in \mathscr{H}_{0}$ are topologized by the induced $C^{\infty}$ uniform topology on their generating vector fields, $X$. That is, a neighborhood base is given by

$$
\begin{aligned}
\mathscr{B}= & \left\{A_{k, \varepsilon}\left(\phi_{t}\right): k \in N, \varepsilon>0, \phi_{t} \in \mathscr{H}_{0}\right\}, \quad \text { where } \\
& A_{k, \varepsilon}\left(\phi_{t}\right)=\left\{\eta_{t} \in \mathscr{H}_{0}:\left\|X-X_{\eta}\right\|_{k}<\varepsilon\right\} .
\end{aligned}
$$


Here $\|\cdot\|_{k}$ denotes the uniform $k$-norm,

$$
\|Y\|_{k}=\max _{\substack{0 \leq j \leq k \\ j=\alpha+\beta}}\left\{\max _{p \in D}\left\|\frac{\partial^{j} Y}{\partial x^{\alpha} \partial y^{\beta}}(p)\right\|\right\} .
$$

Now form the set $\mathscr{H}$ of all $C^{\infty}$-flows, $\phi_{t}$ on $\mathbf{R}^{2}$, for which there exists $\phi_{t} \in \mathscr{H}_{0}$, and a $C^{\infty}$ diffeomorphism $f: \mathbf{R}^{2} \rightarrow \mathbf{R}^{2}$ with $\psi_{t}=f \phi_{t} f^{-1}$. Flows $\psi_{t}$ in $\mathscr{H}$ can be $C^{\infty}$-uniform topologized as have those of $\mathscr{H}_{0}$, by maximizing $k$-derivatives over an associated region, $f(D)$, comparing only those flows having a hyperbolic sector on $f(D)$.

The conjugacy class of a flow in $\mathscr{H}_{0}$ (or in $\mathscr{H}$ ), which is generated by a $C^{\infty}$-flat vector field at $(0,0)$, can be altered either by gluing in a slow linear flow near $(0,0)$, or another $C^{\infty}$-flat flow with dissimilar conjugacy class. For example, those $C^{\infty}$-flat flows which lie outside the central conjugacy class, $I_{0}$, such as the example of [SSW], can be $C^{\infty}$-closely approximated by a $C^{\infty}$-flow which is linear near $(0,0)$. This new flow will have an asymptotically monotone sojourn map, and thus be an element of $I_{0}$. On the other hand, $C^{\infty}$-flat flows within $I_{0}$ can be $C^{\infty}$-closely approximated by a flow having the Shafer-SwansonWalker example near $(0,0)$. In this way, $C^{\infty}$-flat flows are shown to lie on $\partial I_{0}$. Lastly, a specific example of a non- $C^{\infty}$-flat flow, lying on $\partial I_{0}$, will be constructed.

First, a vector field gluing lemma is proven. Let $\beta$ denote the usual bump function such that for $x \leq 0$ and $y \geq 1, \beta(x)=0, \beta(y)=\beta(1)$, and $\beta(x)=\int_{0}^{x} e^{-1 / t^{2}} e^{-1 /(t-1)^{2}} d t$, for $0<x<1$. Next set

$$
M_{j}=\max _{0 \leq x \leq 1}\left\|D^{j} \beta(x)\right\|,
$$

and notice that, $M_{j} \leq M_{j+1}$, for all $j \geq 0$. The bump function $\beta$ may be rescaled to

$$
\beta_{R}(x)=\beta(x / R-1) / \beta(1), \quad \text { for } R>0 .
$$

Then, $\left\|D^{j} \beta_{R}\right\| \leq M_{j} / R^{j}$, for all $j \geq 0$.

LEMMA 5.1. Fix $k \in N$ and $\varepsilon>0$. Let $X$ be a $C^{\infty}$ vector field which is $C^{\infty}$-flat at $(0,0)$. Then there exists $R_{0}=R_{0}(\varepsilon, k)>0$ such that, in polar coordinates, for all $p=(r, \theta)$ with $r<2 R_{0}$,

$$
\left\|\left(1-\beta_{R}\right)(r) \cdot X(p)\right\|_{k} \leq \varepsilon .
$$

Furthermore, there exists $\delta+\delta(\varepsilon, k)>0$ such that, if $L$ is a linear vector field for which $\|L\| \leq \delta$, then

$$
\left\|\left(1-\beta_{R}\right)(r) \cdot L(p)\right\|_{k} \leq \varepsilon .
$$


Proof. Since the $k$-norm is the maximum over the first $k$ derivatives, then for $0 \leq j \leq k$, and $0 \leq r \leq 2 R$,

$$
\begin{aligned}
\left\|D^{j} \beta_{R} X\right\| & \leq\left\|D^{j} \beta_{R}\right\| \cdot\|X\|_{k} \\
& \leq\left\|D^{k} \beta_{R}\right\| \cdot\|X\|_{k} \leq M_{k}\left(\|X\|_{k}\right) / R^{k} .
\end{aligned}
$$

But since $X$ is $C^{\infty}$-flat at $(0,0)$, we must have that $X=o\left(R^{k}\right)$. Thus there exists $R_{0}$ such that $\|X(p)\|_{k} \leq \varepsilon / 2$ on $\left\{r \leq 2 R_{0}\right\}$ and $\|X\|_{k} \leq$ $\left(\varepsilon R_{0}^{k}\right) / 2 M_{k}$, verifying the first inequality. Similarly, since $\|L\|_{k}=$ $\|L\|=\delta$, choose $\delta \leq \min \left(\varepsilon / 2,\left(R_{0}^{k}\right) / M_{k}\right)$ and the Lemma follows.

Proof of Theorem 5.2. First assume that $\phi_{t} \in \mathscr{H}_{0}$ and is generated by a $C^{\infty}$-flat vector field $X \notin I_{0}$. It does not suffice now to bump $L$ off into $X$, using Lemma 5.1. This is because $X$ may have an opposite sense from $L$ at points of $D$ : the newly formed vector field will thus not be an element of $\mathscr{H}_{0}$. Thus, a smooth bumped-off combination of $L$ and $X$ is formed, which respects sense, as follows: For $p \in$ $\left(D \backslash W^{s}(0,0)\right) \backslash W^{u}(0,0)$, let $\bar{p} \in \Sigma_{1}$ and $t(p) \geq 0$ be such that $\phi_{t(p)}(\bar{p})=$ $p$ and $\phi_{t}(\bar{p}) \in D$ for $0 \leq t \leq t(p)$. Then define the "total rotation" in $Y \in \mathscr{H}_{0}$ by

$$
\theta(Y)(p)=\int_{0}^{t(p)} \alpha^{\prime}\left(Y\left(\phi_{t}(\bar{p})\right)\right) d t
$$

where

$$
a(v)=\operatorname{Cos}^{-1}(v \cdot(1,0)) /|v| \text {. }
$$

Notice that $\theta(Y)$ is $C^{\infty}$ on $\left(D \backslash W^{s}(0,0)\right) \backslash W^{u}(0,0)$ and can be $C^{\infty}$ extended to $D \backslash(0,0)$ in the obvious way.

Now fix $k$ and $\varepsilon>0$, and let $Z_{\varepsilon}$ be the vector field on $D$ which has magnitude and sense prescribed by, $\left|Z_{\varepsilon}\right|=\left(1-\beta_{R}\right)|L|+\beta_{R}|X|$ and, off $(0,0), \alpha\left(Z_{\varepsilon}\right)=\left[\left(1-\beta_{R}\right) \theta(L)+\beta_{R} \theta(X)\right] \bmod 2 \pi$, where $R=$ $R_{0}(\varepsilon / 4, k)$ and $\|L\|=\delta(\varepsilon / 4, k)$ as in Lemma 5.1. Then $Z_{\varepsilon}$ is $C^{\infty}$ and $Z_{\varepsilon} \in \mathscr{H}_{0}$ because only $(0,0)$ is a singularity and $Z_{\varepsilon}$ ingresses and egresses properly on $D$.

As constructed, outside the ball $R_{2 R},\left\|Z_{\varepsilon}-X\right\|_{k}=0$ since $Z_{\varepsilon}=X$. Inside $B_{2 R}$,

$$
\left\|Z_{\varepsilon}-X\right\|_{k} \leq 2\left\|\left(1-B_{R}\right) L-\left(1-\beta_{R}\right) X\right\| \leq \varepsilon .
$$

The factor of 2 is required since $\left(1-\beta_{R}\right) L$ and $\beta_{R} X$ may be opposed. Thus, $\phi_{t} \in \mathrm{Cl} I_{0}$ since each $Z_{\varepsilon}$ has a $\tau$-map which is asymptotically monotonic near 0 .

To show that, in fact, $\phi_{t} \in \partial I_{0}$, we select in place of $L$ above a $C^{\infty}$-flat element of $I_{0}^{c}$. The vector field $X_{1}$ of [SSW] has undamped 
time oscillations and is $C^{\infty}$-flat at $(0,0)$. For each $k$ and $\varepsilon>0$, a new $Z_{\varepsilon}$ is formed which equals $X_{1}$ inside $B_{R}$. This is an element of $I_{0}^{c}$ and $\left\|Z_{\varepsilon}-X\right\|_{k}<\varepsilon$. Thus $\phi_{t} \in \partial I_{0}$.

Lastly, a flow $\phi_{t} \in \partial I_{0}$ which is not $C^{\infty}$-flat is constructed. Consider the system

$$
\left\{\begin{array}{l}
x^{\prime}=x\left(x^{2}-y^{2}\right)^{2}+x g(x y) \\
y^{\prime}=-y\left(x^{2}-y^{2}\right)^{2}-y g(x y)
\end{array}\right.
$$

where

$$
g(v)=\left\{\begin{array}{l}
{\left[\exp \left(-|v|^{-1}\right) \sin ^{2}\left(\pi v^{-1}\right)+\exp \left(-v^{-2}\right)\right], \quad v \neq 0} \\
0, \quad v=0
\end{array}\right.
$$

The first quadrant is a hyperbolic sector for (1). We will show that the flow of (1) is not topologically conjugate, in any neighborhood of the origin in the first quadrant, to the standard hyperbolic sector.

Let $u=x^{2}-y^{2}$ and $v=x y$. Then

$$
v^{\prime}=0 \text { and } u^{\prime}=\left(2 u^{2}+2 g(v)\right)\left(x^{2}+y^{2}\right) .
$$

Thus, in $-1 \leq u \leq 1,0<v \leq 1$, and along a solution curve $v=$ constant, we have:

$$
\left(2 u^{2}+2 g(v)\right) 2 v \leq u^{\prime} \leq\left(2 u^{2}+2 g(v)\right)(3) .
$$

For fixed $v, 0<v \leq 1$, let $\tau(v)$ be the time it takes the solution of (1) to pass from $u=x^{2}-y^{2}=-1$ to $u=1$ along the curve $x y=v$. Integrating the inequality (2) we obtain:

$$
\begin{aligned}
& \frac{1}{3}(g(v))^{-1 / 2} \arctan (g(v))^{-1 / 2} \\
& \quad \leq \tau(v) \leq 2 v(g(v))^{1 / 2} \arctan \left(g(v)^{1 / 2}\right) .
\end{aligned}
$$

Now let $v_{n}=2 /(n+1), n=1,2, \ldots$. From (3) and the definition of $g(v)$ we obtain

$$
\begin{aligned}
\tau\left(v_{2 n-1}\right) & \geq(\pi / 12) \exp \left(n^{2}\right) \quad \text { and } \\
\tau\left(v_{2 n}\right) & \leq(((2 n+1) \pi) / 8) \exp (n+(1 / 2)) .
\end{aligned}
$$

Thus, $\tau(1 / n)-\tau(1 /(n+(1 / 2))) \rightarrow \infty$ as $n \rightarrow \infty$. It follows from Proposition 2.2 of [SSW] that the flow of (1) is not topologically conjugate, in any neighborhood of the origin, to the standard hyperbolic sector.

Let $X$ be the vector field given by (1). Then $X$ is not in the standard class $I_{0}$ and $X$ is not $C^{\infty}$-flat. Also, if $L$ is the linear vector field

$$
\left.\begin{array}{l}
x^{\prime}=x \\
y^{\prime}=-y
\end{array}\right\} \quad L
$$


Then $X+\varepsilon L$ is in $I_{0}$ for every $\varepsilon>0$ by Hartman's Theorem. Clearly

$$
X+\varepsilon L \rightarrow X
$$

in the $C^{\infty}$-uniform topology on any compact neighborhood of the origin as $\varepsilon \rightarrow 0$ so $X \in \partial I_{0}$.

Comment. The straight flows mentioned in $\S 1$ can be shown to lie in $\mathrm{Cl}_{0}$ simply by the addition of a small linear vector field.

\section{REFERENCES}

[AGLM] A. A. Andronov, E. A. Leontowich, J. J. Gordon, and A. G. Maier, Qualitative Theory of Second Order Dynamic Systems, (translated from Russian), John Wiley and Sons, 1973.

[D1] Freddy Dumortier, Singularities of vector fields on the plane, J. Differential Equations, 23 (1977), 53-106.

[D2] - Singularities of Vector Fields, Monographs de Matematica, No. 32, IMPA, Rio de Janeiro, 1978.

[GG] Martin Golubitsky and Victor Guillemin, Stable Mappings and their Singularities, Springer Verlag, Graduate Texts in Mathematics No. 14, New York, 1973.

[H] P. Hartman, Ordinary Differential Equations, second edition, Birkhauser, Boston, 1982.

[SSW] Douglas Shafer, Richard Swanson, and Russell Walker, Topological conjugacy of singularities of vector fields, Topology, 25 (1986), 521-539.

Received January 15, 1988 and in revised form August 15, 1988.

Montana State University

BOZEMAN, MT 59717 



\section{PACIFIC JOURNAL OF MATHEMATICS EDITORS}

\author{
V. S. VARAdarajan \\ (Managing Editor) \\ University of California \\ Los Angeles, CA 90024-1555-05 \\ Herbert Clemens \\ University of Utah \\ Salt Lake City, UT 84112 \\ ThOMAS ENRIGHT \\ University of California, San Diego \\ La Jolla, CA 92093
}

R. FINN

Stanford University

Stanford, CA 94305

HeRmann FlaschKa

University of Arizona

Tucson, AZ 85721

VAUGHAN F. R. Jones

University of California

Berkeley, CA 94720

SteVen KercKhofF

Stanford University

Stanford, CA 94305

\section{ROBION KIRBY}

University of California

Berkeley, CA 94720

C. C. MOORE

University of California

Berkeley, CA 94720

HAROLD STARK

University of California, San Diego

La Jolla, CA 92093

\section{ASSOCIATE EDITORS}
R. Arens
E. F. BeCKenbaCH
B. H. NEUMANN
F. Wolf
K. YoshidA (1906-1982)

\section{SUPPORTING INSTITUTIONS}

UNIVERSITY OF ARIZONA

UNIVERSITY OF BRITISH COLUMBIA

CALIFORNIA INSTITUTE OF TECHNOLOGY

UNIVERSITY OF CALIFORNIA

MONTANA STATE UNIVERSITY

UNIVERSITY OF NEVADA, RENO

NEW MEXICO STATE UNIVERSITY OREGON STATE UNIVERSITY

\author{
UNIVERSITY OF OREGON \\ UNIVERSITY OF SOUTHERN CALIFORNIA \\ STANFORD UNIVERSITY \\ UNIVERSITY OF HAWAII \\ UNIVERSITY OF TOKYO \\ UNIVERSITY OF UTAH \\ WASHINGTON STATE UNIVERSITY \\ UNIVERSITY OF WASHINGTON
}

The Supporting Institutions listed above contribute to the cost of publication of this Journal, but they are not owners or publishers and have no responsibility for its content or policies.

Mathematical papers intended for publication in the Pacific Journal of Mathematics should be in typed form or offset-reproduced (not dittoed), double spaced with large margins. Please do not use built up fractions in the text of the manuscript. However, you may use them in the displayed equations. Underline Greek letters in red, German in green, and script in blue. The first paragraph must be capable of being used separately as a synopsis of the entire paper. In particular it should contain no bibliographic references. Please propose a heading for the odd numbered pages of less than 35 characters. Manuscripts, in triplicate, may be sent to any one of the editors. Please classify according to the scheme of Math. Reviews, Index to Vol. 39. Supply name and address of author to whom proofs should be sent. All other communications should be addressed to the managing editor, or Elaine Barth, University of California, Los Angeles, California 90024-1555-05.

There are page-charges associated with articles appearing in the Pacific Journal of Mathematics. These charges are expected to be paid by the author's University, Government Agency or Company. If the author or authors do not have access to such Institutional support these charges are waived. Single authors will receive 50 free reprints; joint authors will receive a total of 100 free reprints. Additional copies may be obtained at cost in multiples of 50 .

The Pacific Journal of Mathematics is issued monthly as of January 1966. Regular subscription rate: $\$ 190.00$ a year (5 Vols., 10 issues). Special rate: $\$ 95.00$ a year to individual members of supporting institutions.

Subscriptions, orders for numbers issued in the last three calendar years, and changes of address should be sent to Pacific Journal of Mathematics, P.O. Box 969, Carmel Valley, CA 93924, U.S.A. Old back numbers obtainable from Kraus Periodicals Co., Route 100, Millwood, NY 10546.

The Pacific Journal of Mathematics at P.O. Box 969, Carmel Valley, CA 93924 (ISSN 0030-8730) publishes 5 volumes per year. Application to mail at Second-class postage rates is pending at Carmel Valley, California, and additional mailing offices. Postmaster: send address changes to Pacific Journal of Mathematics, P.O. Box 969, Carmel Valley, CA 93924.

PUBLISHED BY PACIFIC JOURNAL OF MATHEMATICS, A NON-PROFIT CORPORATION Copyright (C) 1989 by Pacific Journal of Mathematics 


\section{Pacific Journal of Mathematics \\ Vol. 140, No. $2 \quad$ October, 1989}

Edoardo Ballico, Spanned and ample vector bundles with low Chern numbers

Marcy Mason Barge, Richard Swanson and Russell Bruce Walker,

Conjugacy class structure of smooth hyperbolic sectors . ........... 217

Jeffrey Stephen Fox, Adeles and the spectrum of compact nilmanifolds . . ..233

Robert D. Little, Homotopy complex projective spaces with divisible

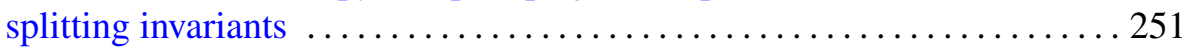

M. Scott Osborne and Garth William Warner, Jr., The Selberg trace formula. VII. Application of the truncation process to the continuous

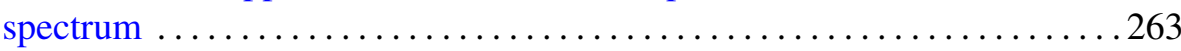

John R. Stembridge, On the eigenvalues of representations of reflection

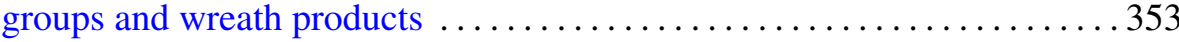

Ibrahim Salama, Corrections to: "Topological entropy and recurrence of

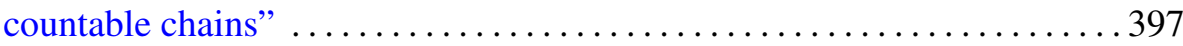

Robert Greene and Hung-Hsi Wu, Addendum to: "Lipschitz convergence of Riemannian manifolds" ............................... 398

Kayoko Shikishima-Tsuji, Correction to: "Galois theory of differential fields of positive characteristic" 